\title{
Analysis algorithm for surface crack detection by thermography with UV light excitation
}

\author{
by P. Broberg* and A. Runnemalm*
}

\author{
* University West, University West, SE-461 86 Trollhättan, Sweden, patrik.broberg@hv.se
}

\begin{abstract}
Surface crack defects can be detected by IR thermography due to the high absorption of energy within the crack cavity. It is often difficult to detect the defect in the raw data, since the signal easily drowns in the background. It is therefore important to have good analysis algorithms that can reduce the background and enhance the defect. Here an analysis algorithm is presented which significantly increases the signal to noise ratio of the defects and reduces the image sequence from the camera to one image.
\end{abstract}

\section{Introduction}

It has previously been shown that surface breaking cracks, in metal structures, can be detected by monitoring the temperature increase in the surface when it is radiated with high intensity light [1]. The method works due to the difference in absorbance of radiation in a smooth metal surface and surface breaking defect, such as a crack. Since a metallic surface generally has a very low absorption coefficient for wavelengths in and around the visual and infrared range, it will reflect most of the incoming light $[2,3,4]$. Light entering an open cavity, such as a crack, on the other hand will be reflected multiple times and will therefore deposit more energy. This results in a higher temperature increase in cracks than in the surrounding surface. The method originally used a pulsed light source for the excitation [1], in order to avoid that the excitation source disturbs the measurement with reflected radiation. Recently, longer square pulses and close to continuous light sources have been used [5,6] with wavelengths not visible to the infrared camera. The wavelength of the light was in this case in the ultraviolet region, in order to improve the absorption and avoid disturbing the measurement with infrared light that is reflected in the surface.

In thermography it is often difficult to detect defects in the raw data, since the signal easily drowns in the background and the signal to noise ratio can be very low. It is therefore important to have good analysis algorithms that can reduce the background and enhance the signal from the defects. Since the temperature increase in the test sample is relatively low, the need for a good analysis method is even more important. Another common goal for many analysis algorithms is to make the defect detection easy for an operator by reducing the image sequence to a single image.

In this paper an analysis algorithm is presented which significantly increases the signal to noise ratio of the defects and combine the data in the image sequence from the camera into one single image.

\section{Theory}

The analysis method proposed is based on the fact that a defect open to the surface absorbs more energy than the surrounding, defect free, surface. The assumption is made that the test piece can be regarded as semi-infinite for the heat conduction, at the relatively short excitation times and low energies used here, and that the illumination is even for the whole area of interest. The heating of the defect area is also assumed to be the same as for the surrounding surface, except for the amount of absorbed energy. The 1-dimentional heat equation, with no heat source is

$$
\frac{\partial T}{\partial t}=\alpha \frac{\partial^{2} T}{\partial x^{2}}
$$

where

$$
\alpha=\frac{k}{c_{p} \rho}
$$

and $k$ is the thermal conductivity, $\rho$ the density, $c_{p}$ the specific heat capacity and $T$ the temperature.

The solution to Eq. (1), with the assumptions given and the initial surface temperature $T_{0}$, gives the temperature of the surface during the cooling as 


$$
T_{C}=\frac{T_{0}}{\sqrt{4 \pi \alpha t}}
$$

This equation gives the characteristic $1 / \sqrt{t}$ curve that can be seen in many pulsed thermography results. Even though the heating is not transient the cooling sequence will approximately follow this curve if the heating is short enough, as can be seen in the experimental results in figure 1. Eq. (1) can be simplified to

$$
T_{C}=k_{C} t^{0.5}
$$

if all constant terms are included in the constant $k_{c}$. This is the equation that will be used in the algorithm presented below. During the heating of the test piece the heat equation has a constant heat source $Q$ at the surface, which means that Eq. (1) will transform to

$$
\frac{\partial T}{\partial t}=\alpha \frac{\partial^{2} T}{\partial x^{2}}+\frac{Q}{c_{p} \rho}
$$

This equation is more demanding to solve analytically and will not give a simple solution that is needed for computational reasons. It is therefore convenient to use an empirical relationship for the surface temperature, $T_{H}$, during the heating. Experimentally it has been noted that the relation can be written as

$$
T_{H}=k_{H} t^{D}
$$

under the conditions used here. Where $k_{H}$ is dependent on the absorbed energy and $D$ is dependent on material properties and excitation time (in relation to the thickness of the plate). $D$ has been seen experimentally to have a value between 0.3 and 0.5 , often closer to 0.5 . Figure 1 shows a typical heating and cooling curve for a defect with Eq. (4) fitted to the cooling curve and Eq. (6), with $D=0.5$, fitted to the heating curve.

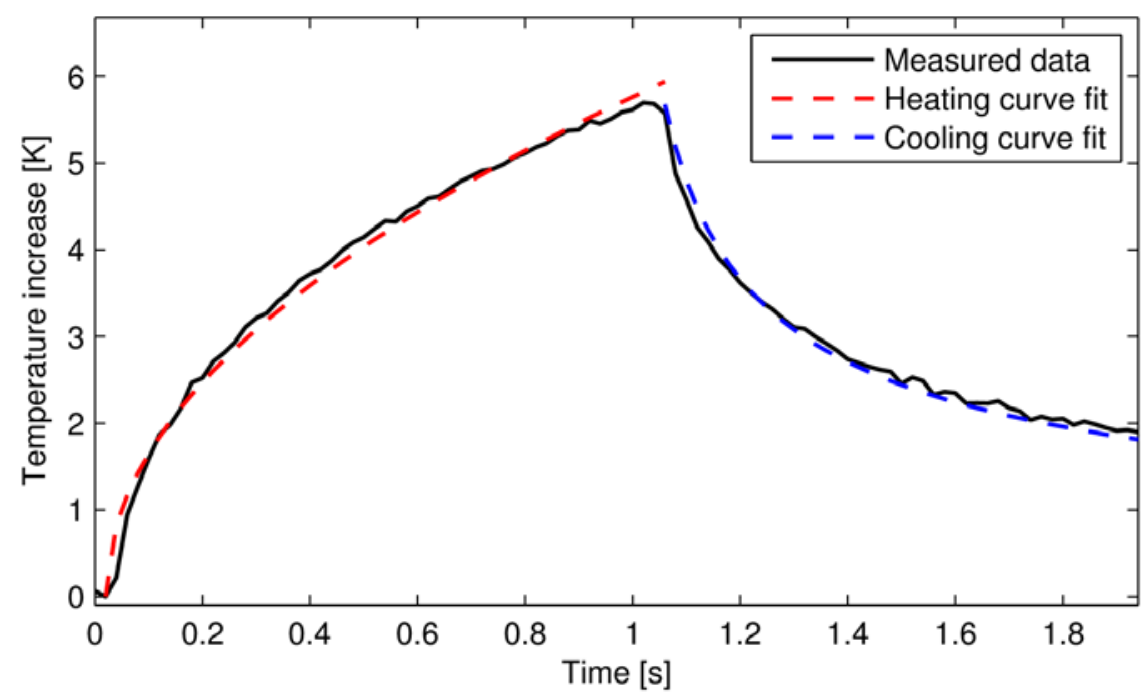

Fig. 1. Measured data from a defect with curves fitted to the heating and cooling

\section{Analysis method}

The heating and cooling of the defect, as well as in the area close to the defect, during the measurement can be seen in figure $2 \mathrm{a}$. The heating and cooling behaviour follow the same curve shape even if the excitation time is increased, within reasonable limits. There is also no noticeable difference between the defect and the background when it comes to the heating and the cooling, except for the amplitude. The goal of the analysis algorithm is to get a measure 


\subsection{1/qirt.2016.014}

of the absorbed energy, in every point in the image, while at the same time reducing the noise and removing the background. Using the maximum value of every pixel is generally not an optimal solution since it is sensitive to transient noise. Instead the whole time sequence is used in order to have a measure of the absorbed energy. This is done by fitting Eq. (4) to the cooling sequence or Eq. (6) to the heating sequence and identifying the value of $k_{c}$ or $k_{H}$ for every pixel in the data set. One example of this, for the heating sequence in a defect, can be seen in figure $2 \mathrm{~b}$. The advantage of this procedure is that it is less sensitive to noise, removes the background, and in addition it does not require a high frame rate of the camera in order to have good results. The result of this algorithm is that the whole image sequence is combined into one single image, while still preserving the useful information. The reason why these two, simplified, models of the heating and cooling is used and not more detailed models, is in order to reduce the computational complexity of the analysis and at the same time the calculation time.

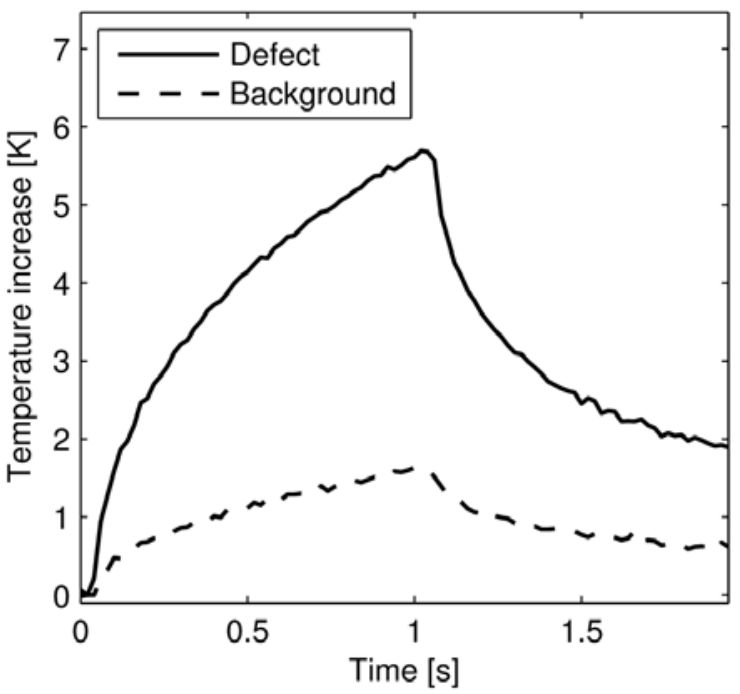

a) Heating and cooling of defect

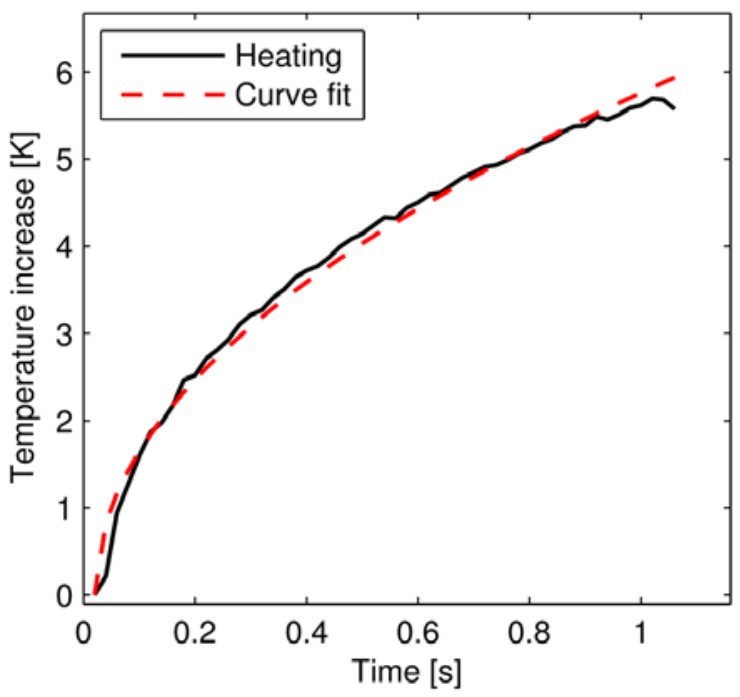

b) Heating of defect with fitted curve

Fig. 2. Temperature increase and decrease in the defect during the measurement.

Since a local heating, such as in a defect, diffuses with time, the heating sequence generally gives a less blurry image than the cooling sequence since the heat has not had time to spread out spatially. The parameter $D$ of the heating curve fit in Eq. (6) is dependent, among other things, on the material. In the figure above the value $D=0.5$ was used. Using the correct value of $D$ is not crucial for good results as long as the difference between the defect and the surrounding, sound, area is large enough.

The procedure for calculating the values of $k_{c}$ and $k_{H}$ is as follows. First the start of the heating, $t_{s t a r t}$, is determined from the data set. This is easies done by finding when the temperature starts to increase in an area of the image that is heated by the excitation source. The time when the heating ends, $t_{e n d}$, is then determined in a similar way. Using these values, a vector, $t_{H}$, starting at zero, with the same sampling frequency as the camera and the same amount of values as there is between $t_{\text {start }}$ and $t_{\text {end }}$ can then be calculated. An equivalent vector, $t_{c}$, from $t_{\text {end }}$ to the end of the recording can also be calculated, starting from zero here as well. In order to avoid division by zero for the first value in the curve fit, a short delay should be added to the times in the vector. With this done it is possible to estimate $k_{c}$ and $k_{H}$ for every pixel in the image set. For every pixel a signal, $T$, is determined with the temperatures from the start of the heating to the end of the recording. The offset temperature is then removed from the signal by subtracting the temperature at the start of the heating, $T-T\left(t_{s t a r}\right)$. The values $k_{c}$ and $k_{H}$ from Eq. (4) and (6) can then be calculated by a least-square curve fitting of Eq. (4) and (6) to the signal. The resulting image is given by plotting $k_{c}$ or $k_{H}$ for every pixel in the image set as one image. A block diagram of the complete analysis method can be seen in figure 3. 


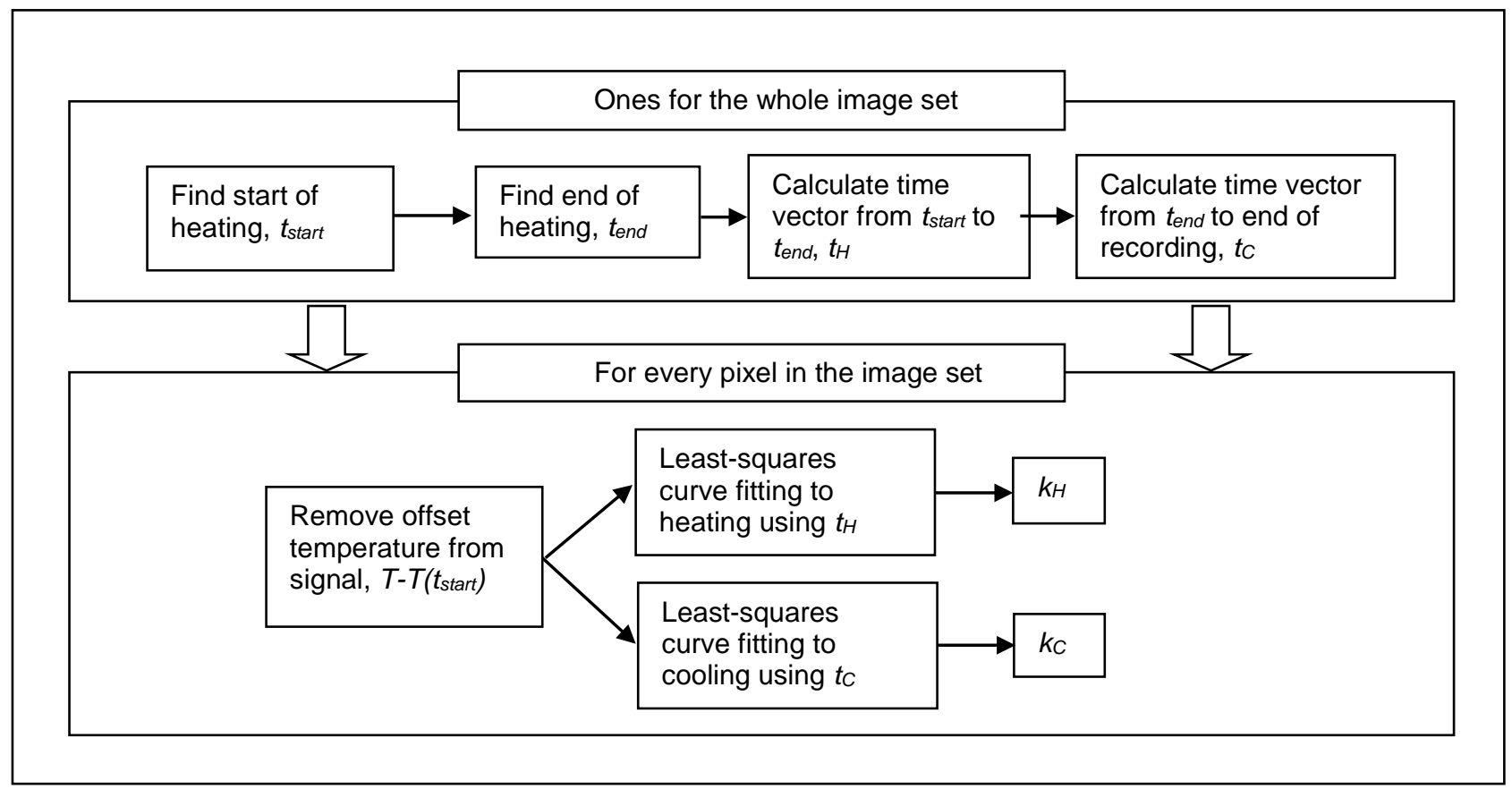

Fig. 3. Block diagram of the analysis method.

\section{Experimental setup}

In order to test the analysis method presented above an experiment was conducted, with a set-up as shown in figure 4. A welded Ti-6-4 plate was used as a test sample. The weld contained several notches, manufactured using electric discharge machining, with lengths from $0.25 \mathrm{~mm}$ to $1.5 \mathrm{~mm}$, width about $0.1 \mathrm{~mm}$ and the depth half of the length. The infrared camera used for the experiments was a FLIR SC 5650 infrared camera with a spectral sensitivity between $2.5-5.1 \mu \mathrm{m}$. The camera had an InSb detector with a resolution of $640 \times 512$ pixels and the frame rate during the recording was 50 images/s. An ultraviolet (UV) light source (Lumatec SUPERLITE I 07) was used as an excitation source. The light source was fitted with a light guide and filters, to guide the light to the test piece and remove light in wavelengths other than in the UV range. The distance between the camera and the test piece was about $200 \mathrm{~mm}$ and the distance between the light source and the test piece around $10 \mathrm{~mm}$. The excitation time used here was 1 second.

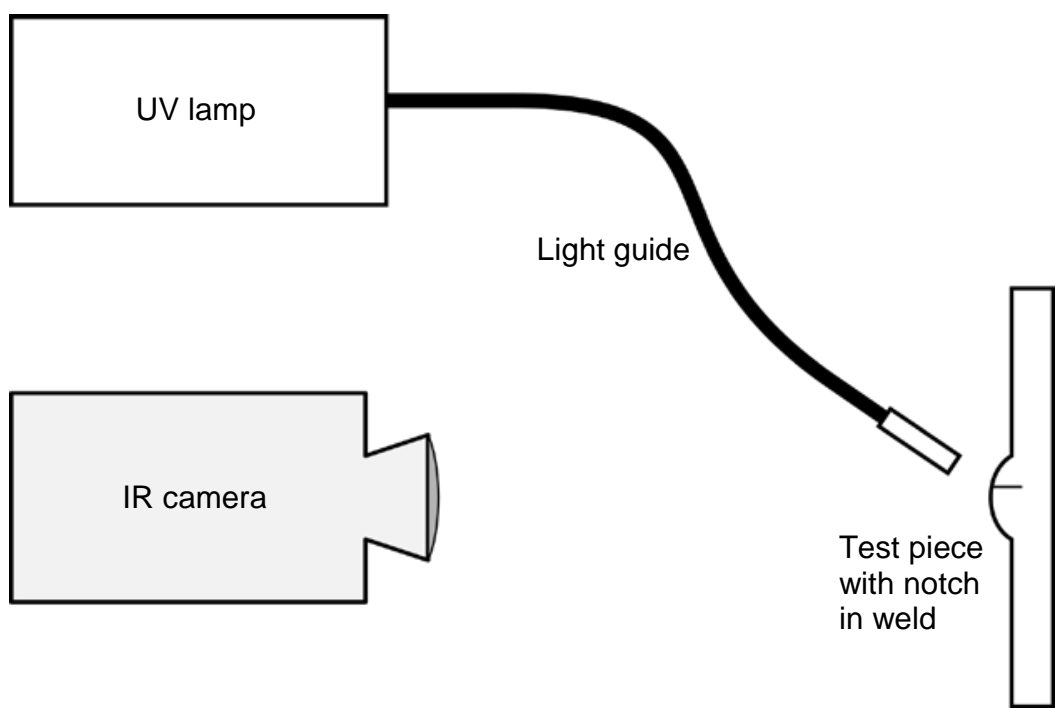

Fig. 4. Experimental setup with IR camera, UV lamp with light guide and a test piece with a notch in a weld. 


\subsection{1/qirt.2016.014}

\section{Results and discussion}

One image from the image sequence, at the end of the excitation time, with a $0.5 \mathrm{~mm}$ long, $0.1 \mathrm{~mm}$ wide and $0.25 \mathrm{~mm}$ deep notch can be seen in figure $5 \mathrm{a}$. The notch is here located in the root of the weld. The notch is heated to an apparent temperature increase of less than $6^{\circ} \mathrm{C}$ and the background to less than $2^{\circ} \mathrm{C}$. Due to the variation in emissivity between the notch and the background, and between different areas in the background, the notch can be difficult to detect even though there is a difference in temperature. In figure $5 b$ the value $k_{H}$ for every pixel is plotted as an image. It can be noted that the signal strength is increased and the background noise is decreased compared to the raw data. It is worth noting that the background is greatly reduced, as is the strong reflections in the weld since they do not change with time. This is important for an operator or automatic program that will evaluate the results since it decreases the chance of false positives.

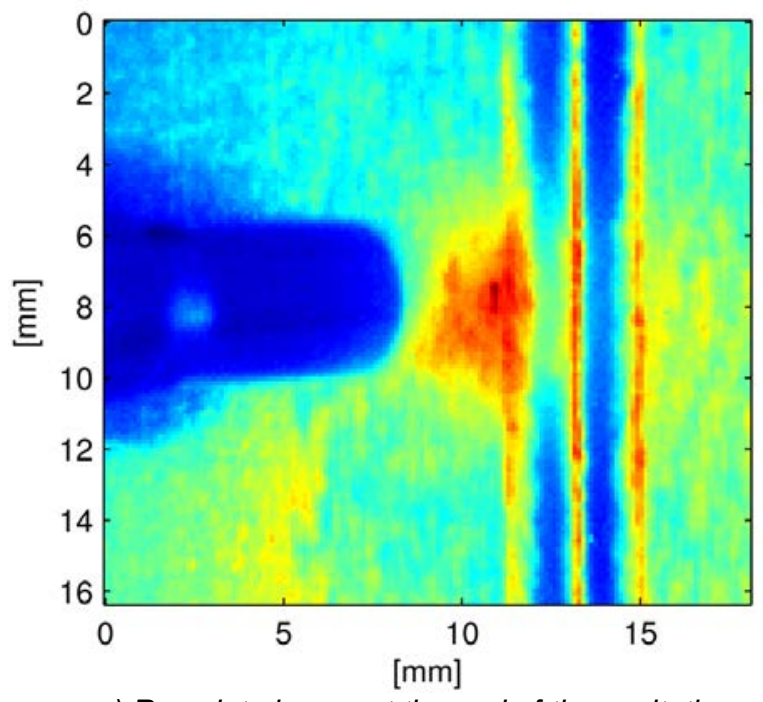

a) Raw data image at the end of the excitation

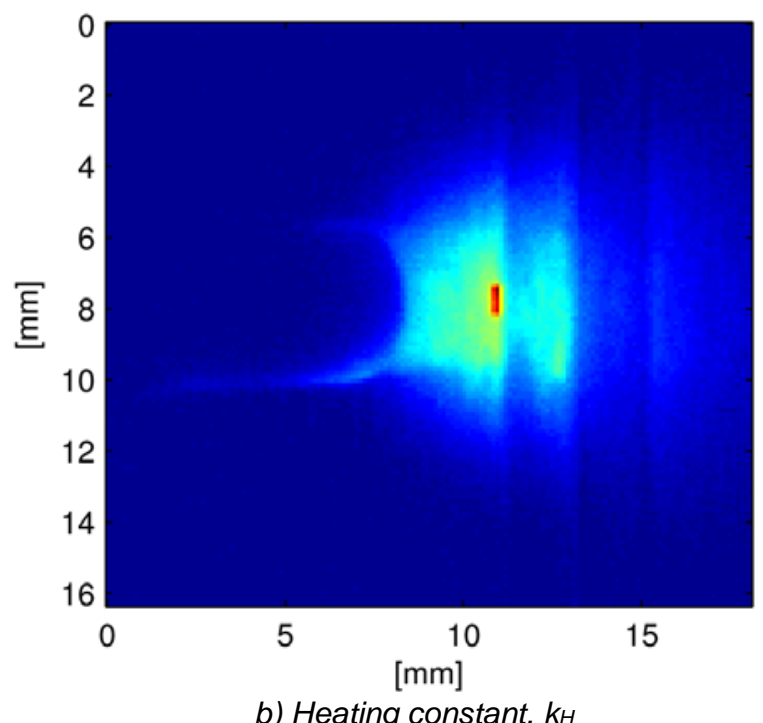

b) Heating constant, $\mathrm{kH}_{\mathrm{H}}$

Fig. 5. Notch in the raw data and in the final results.

The signal to noise ratio (SNR) was calculated using

$$
S N R=\frac{\mu_{\text {Defect }}}{\sigma_{\text {Background }}}
$$

for both the raw data, at the end of the heating, as well as for the resulting image after the analysis. Here $\mu_{\text {Defect }}$ is the average signal strength from a defect with the average background level subtracted, and $\sigma_{\text {Background }}$ is the standard deviation of the background. The SNR for both the $k_{c}$ and the $k_{H}$ image was calculated and compared with SNR in the raw data, see table 1 . The SNR value during the cooling, when $k_{c}$ is used, is lower than during heating, when $k_{H}$ is used, since the signal for the defect becomes less well defined during cooling. In addition the background level increases since it reflects the heat in the light source, which will be higher for the cooled down surface, due to the fact that the cooling rate in the light source is less than in the test sample.

Table 1. SNR values for the raw data and the resulting images.

\begin{tabular}{|c|c|c|}
\hline SNR raw data & SNR cooling, $k_{c}$ & SNR heating, $k_{H}$ \\
\hline 2.6 & 6.2 & 7.2 \\
\hline
\end{tabular}




\subsection{1/qirt.2016.014}

\section{Conclusions}

Surface crack defects can be detected by IR thermography due to the high absorption of energy within the crack cavity. With this thermography method it is often difficult to detect the defect in the raw data, since the temperature increase is small and the signal easily drowns in the background. It is therefore important to have good analysis algorithms that can reduce the background and enhance the defect signal. Here an analysis algorithm has been presented that significantly increases the signal to noise ratio of the defect as well as reduce the background and the influence of transient noise. The algorithm also combines all images in the image sequence from the camera to one single image, which makes the results easier to handle for an operator or a program for automatic defect detection. It was shown that the results from the heating sequence resulted in a higher SNR value than for the results from the cooling sequence. This is due to the fact that the defect signal becomes more blurry with time and the background level increases since the reflections from the relatively warmer excitation source become more dominant.

\section{REFERENCES}

[1] Broberg P., "Surface crack detection in welds using thermography", NDT \& E International, vol. 57, pp. 69-73, 2013.

[2] Bergström D., Powell P., Kaplan A. F. H., "A ray-tracing analysis of the absorption of light by smooth and rough metal surfaces", Journal of Applied Physics, vol. 101, pp. 1-11, 2007.

[3] Bergström D., Kaplan, A., "Mathematical modelling of laser absorption mechanisms in metals: A review", presented at the 16th Meeting on Mathematical Modeling of Material Processing with Laser, Igls, Austria, 2003.

[4] Prokhorov, A. M., Konov, V. I., Ursu, I., Mihailescu, I. N., "Laser heating of metals". Bristol, England: Adam Hilger, 1990.

[5] Runnemalm A., Broberg P., "Surface crack detection using infrared thermography and ultraviolet excitation," presented at the 12th International Conference on Quantitative InfraRed Thermography, Bordeaux, France, 2014.

[6] Runnemalm A., Broberg P., Henrikson, P., "Ultraviolet excitation for thermography inspection of surface cracks in metal structures," Nondestructive Testing and Evaluation, vol. 29, pp. 332-344, 2014. 